\title{
CHANGES IN EMOTIONAL FUNCTIONING IN WOMEN WITH CARCINOMA OF THE MAMMARY GLAND
}

\author{
Silviya Tzvetkova ${ }^{1}$, Kameliya Tsvetanova $^{2}$, Ivelina Ivanova ${ }^{3}$, Slavcho Tomov ${ }^{4}$, \\ Todor Dimitrov ${ }^{5}$
}

${ }^{1}$ Faculty of Public Health, Medical University - Pleven

${ }^{2}$ Clinic of Anaesthesiology and Intensive Care, UMHAT "Dr. G. Stranski" Ltd. - Pleven

${ }^{3}$ Department of Medical Oncology, UMHAT “Dr. G. Stranski” Ltd. - Pleven

${ }^{4}$ Medical University - Pleven

${ }^{5}$ Clinic of Gynecologic Oncology, UMHAT “Dr. G. Stranski” Ltd. - Pleven

\begin{abstract}
The carcinoma of the mammary gland is the most frequent female malignant disease in Bulgaria and in most of the developed countries. Its share is about $25 \%$ of all malignant diseases and it takes a second place among the mortality reasons due to oncological diseases. The cancer disease inevitably leaves a trace in women and changes their quality of life, influences their psychosocial status, causes anxiety and distress and changes their views on life and the future.

All the spheres building the concept „quality of life” are influenced in different ways by the dynamics of emotionality and experience.

The current research study examines the correlation between subjectively estimated anxiety which is proved by tools and psychosocial functioning in women with breast cancer.
\end{abstract}

Keywords: carcinoma of mammary gland, anxiety, quality of life

\section{INTRODUCTION}

The carcinoma of mammary gland is the most frequent female malignant disease in Bulgaria and in most of the developed countries. Its share is about $25 \%$ of all malignant diseases and it takes a second place among the mortality reasons due to oncological diseases.

Address for correspondence:

Kameliya Todorova Tsvetanova

Clinic of Anaesthesiology, Reanimation and Intensive Care,

Oncology Center, University Hospital - Pleven, 8A,

Georgi Kochev, blvd Pleven, 5800 Bulgaria

e-mail: kamelia_tsvetanova@abv.bg

Received: September 16, 2016

Accepted: December 21, 2016
According to data of the World Health Organization from 2010, the newly diagnosed cases of patients with breast cancer are 1500000 and 411000 people died due to the fatal disease.

Morbidity varies between the countries in the world. The difference between the lowest (Mozambique) and the highest indicators (USA) is around 25 times (1).

Every $22^{\text {nd }}$ woman in Bulgaria can fall ill from such a disease (2). According to data from 2011, 3885 new cases of breast cancer were registered in Bulgaria and the deaths for the female population were 1284 . Every year the morbidity rate of carcinoma of mammary gland increases with $1.8 \%$ on average.

Morbidity rates increase with the progress in age of ill people and this increase can be observed in all age groups. The disease affects mostly women 
Silviya Tzvetkova, Kameliya Tsvetanova, Ivelina Ivanova et al.

over 45 years of age and reaches its peak among those aged 60-64 years.

About 3/4 of the patients (or 71.2\%) are diagnosed in the opening stage ( $1^{\text {st }}$ and $2^{\text {nd }}$ stage) of the disease progress. $26 \%$ are diagnosed in 3rd and 4th stage and the stage is not specified in the rest of the cases.

The standardized morbidity of breast cancer in Bulgaria is lower than the mean value for Europe. Forecast data for 2012 indicate morbidity rate for the country of 76.3 cases out of 100000 women as the average rate for Europe is 94.2 cases out of 100000 . However, the standardized mortality rate due to cancer of mammary gland in Bulgaria is higher than the average for Europe - 24.1 and 23.1 out of 100000 women, respectively.

The relative five-year survival period for mammary gland cancer is $71.7 \%$ in Bulgaria. It is lower than the average for Europe where it is $81.8 \%$ (2).

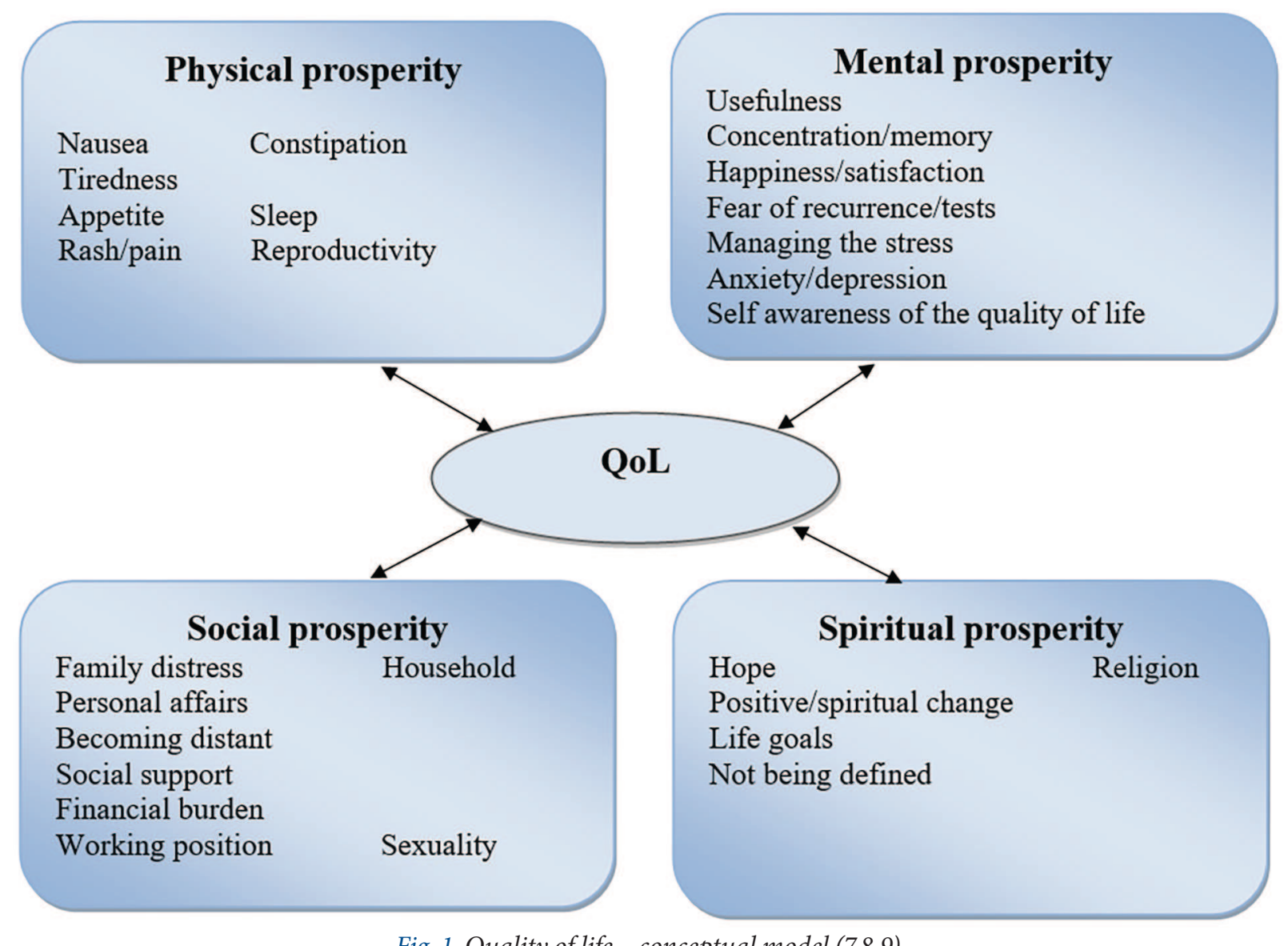

Fig. 1. Quality of life - conceptual model $(7,8,9)$
Quality of Life of Women Diagnosed with Breast Cancer

Quality of life is a concept which is affected by factors like: physical health of persons, their psychological condition and level of independency, social relationships and their links to the significant characteristics of their environment. The quality of life related to health is a multidimensional concept including physical, psychological, spiritual and social prosperity of individuals. It is directed towards the personality and its psychological and social aspects, to its social role in society, emotional condition, mutual relations with other people and satisfaction from activities performed. The idea of quality of life consists not only of the condition of personality but it includes also the degree of expectations and dreams reflected in everyday life.

It is proven that the quality of life changes in the various periods of life. The more advanced the person's age is and the more life experience accumulate there is, the easier one can adapt to his/her health

Usefulness

Concentration/memory

Happiness/satisfaction

Fear of recurrence/tests

Managing the stress

Anxiety/depression

Self awareness of the quality of life 
condition. Younger people have higher expectations regarding their physical and functional condition. According to some authors $(3,4)$, the quality of life is higher in elder people compared to younger ones. Quality of life is multidimensional term including different spheres of life, any one of them with different significance attached. 12 to 14 different spheres are described (5) which proves the complex nature of the concept examined (6). The multicomponent pattern for quality of life, presented in Fig. 1, illustrates the physical, mental, spiritual and social prosperity of patients with oncological diagnosis (6) in a meaningful aspect.

\section{$\diamond$ Physical prosperity (6)}

Physical prosperity is connected with the control or relief of the symptoms and the ability of the patient to self-handle and operate independently. In women with breast cancer physical prosperity is disturbed in a series of aspects. These problems can be connected with reproduction, sleep, pain, lymph oedema of the hand from the side of the affected breast, premature menopause due to continuous hormonal therapy and chemotherapy, difficulties in performing of usual physical exercises and daily activities, accumulating of excess weight, sexual problems, etc.

\section{$\diamond$ Mental prosperity (6)}

Mental prosperity is associated with the desire to control the diagnosis of breast cancer which diagnosis is determined by emotional distress, change of life priorities and values, fear of the unknown. A number of studies (10) which cover the period immediately after diagnosing till the completing of therapy demonstrate that the initial distress gradually decreases over time and the female patients reach a relative stability in emotional perspective. Women suffering from cancer improve their quality of life (11) at the end of the first year after diagnosing. Other studies (12) prove that approximately $1 / 3$ of the patients with breast cancer live with depression symptoms which are reduced in the course of time. Many women with cancer resort to using alcohol or drugs trying to reduce the stress, anxiety, nervousness and sadness (13). Frequent fears can be connected with their future; they can lose faith, significance and goals in life.

The main psychosocial fears (11) they share are as follows (14): $\diamond$ fear of recurrence

$\diamond$ fatigue, sleep problems, pain

$\diamond$ own body perception difficulties

$\diamond$ sexual dysfunctions

$\diamond$ anxiety related to treatment

$\diamond$ intrusive thoughts about the disease or permanent anxiety

$\diamond$ communication problems with partner

$\diamond$ a feeling of vulnerability

$\diamond$ existential issues related to their own death

The emotional sphere takes a significant place in the theme for mental prosperity of the cancer patients. One of the most frequent changes are high sensitivity and low frustration threshold. Processing of any information related to the disease and its results is

$\diamond$ Social prosperity $(7)$

Social prosperity represents the ability to cope successfully with disease consequences which the cancer experts on personality and its social and interpersonal relations. In other words, it is the ability of the patient to defend his/her position and role in the society. According to a research made (13), many of the women diagnosed are afraid of being discriminated and underestimated by their colleagues and bosses and they worry about their career growth. Another problem found in breast cancer operated women is connected with their sexual functioning and disrupted intimacy with their partners. Often problems emerge exactly because of the perception of their own body and building inferiority complex. Many of the female patients suffer torments about their children and future, parenthood, financial burdens, etc. (13).

\section{$\diamond$ Spiritual prosperity (6)}

Spiritual prosperity is the ability to maintain hope and to draw a lesson from oncological experience. This experience can possibly have a positive psychological and social impact on women with breast cancer. According to research data (15) aiming to prove this positive influence, many of the women share that after the diagnosis of this fatal disease and passing through the different stages of its treatment, they start to appreciate life more, pay less attention to the daily chores, decide to live according to the prin- 
Silviya Tzvetkova, Kameliya Tsvetanova, Ivelina Ivanova et al.

ciple 'here and now' and pay less attention to the material matters appreciating the spiritual ones more.

According to other research studies (16), younger women suffer deeper pain, accept the disease harder and their quality of life is lower after the severe diagnosis - cancer of the mammary gland. Young patients are frequently tormented by doubts if they will have children. The reason is their premature menopause as a consequence of the hormonal therapy lasting at least 5 years and leading to a loss of reproductive capabilities. The symptoms of premature menopause, sexual problems and the problem with the perception of their own body generate anxiety, too.

Negative changes occur in sexuality, frequent episodes of fatigue appear, distress comes on and continuous questions about the future arise after diagnosing the cancer of mammary gland. Positive changes occur in life priorities and spirituality of female patients. Part of women suffer overweight and symptoms like vaginal dryness, night sweats, warm waves, changes in mood etc. that disturb the quality of their life.

A number of studies are conducted $(14,17)$ about the impact of relapses, i.e. recurrence of the disease. Many of the female patients share that they lose hope, accept their own health in a more negative light, less and less they trust medicine and the doctors treating them (18)

Social and family factors such as family closeness and support also influence the quality of life of the patient. Family solidarity represents the inner strength of the family to overcome difficulties and changes. It is connected indirectly with higher quality of life of women with breast cancer (6).

Besides the aspects pointed out, the disease represents a large financial and economic burden for patients and members of their families $(6,19)$. Expenses resulting from the diagnosis are related to consultations with doctors, perpetual medical examinations or complementary therapy. Costs for clothing and change of appearance increase also, being a consequence of the operation performed and the follow-up treatment, as well as expenses for food additives, immunostimulants etc. The combination of additional expenses and reduced income provokes new fears, anxiety and distress in patients.
The quality of life related to health is determined by the impact of the diagnosis on patients. There is a variety of tools for measurement of the impact as most of the questionnaires are worked out with the objective to access three aspects of prosperity: physical, emotional and social. Normally, different questionnaires are used for filling in by patients (6). As we have noted already, the quality of life which is connected with health is a multidimensional term. We use different sub-scales for its measurement which indicate different aspects. Summarized questions are used also for calculation of the mean value of the quality of life in case of cancer of mammary gland.

\section{MISSION AND OBJECTIVES}

The main objective of the research is to establish the impact of the disease process on physical and psychosocial functioning in women with cancer of the mammary gland. The specific objectives related to the topic are connected with an explanation of changes in the emotional sphere in women with breast cancer with a focus on anxiety.

\section{METHODOLOGY AND MATERIALS}

The object of the study is women with carcinoma of mammary gland.

The subject of the study is dependencies and correlations between the levels of anxiety and the spheres which determine quality of life of the patients inquired.

Place of conducting the study: Oncological centre at Dr G. Stranski University Hospital - Pleven

\section{Patients Studied}

37 women diagnosed with cancer of the mammary gland are examined at random. The method used is direct individual inquiry.

\section{METHODS}

The objective of the study requires using the sociological method - inquiry and statistical methods. The methods used give us the possibility to establish the main characteristics of women with cancer disease, the leading problems caused by changes in the quality of life, the level of anxiety and distress. The study is voluntary and anonymous as the patients have received personal information about the objectives and the character of inquiry. 
The following materials are used:

$\diamond$ Freely structured inquiry containing demographical data

$\diamond$ Scale of Taylor for assessment of the general level of anxiety

The Questionnaire TMAS (Taylor Manifest Anxiety Scale) was developed by J. Taylor and it measures the level of anxiety as a property of the personality (20). The questionnaire contains 50 questions.

$\diamond$ Distress thermometer - it is a combined methodology. Emotional changes are commented in the material.

$\diamond$ Questionnaire about the quality of life (Questionnaire-Core 30 of the European Organization for Research and Treatment of Cancer / EORTC/ QLQ-C30). EORTC QLQ-C30 is used to assess the quality of life in cancer patients. Breast cancer module: QLQ-BR23 is applied to the first part. The emotional operation is highlighted which is the meaningful part of the functional scales.

$\diamond$ Excel and StatGraf (Multiple-Variable Analysis, Spearman Rang Correlations; Crostabulation Analysis) programmes are used for statistical processing of the primary results.

\section{Analysis of the Demographic Indicators}

37 women with proven cancer take part in the study. The age of the women inquired is between 29 and 69 years and the average age is 53.86 years.

According to the division by age (Fig. 2), 2 women (or 5\%) belong to the group 18 to 35 years. 10 (or 27\%) belong to the group 35 to 50 years old. 23 (or 62\%) of the patients inquired belong to the 50-65 years age group. 2 (or 5\%) of the participants in the study belong to the group over 65 years.

The data indicate clearly that the most affected group, representing over a half of the patients in the

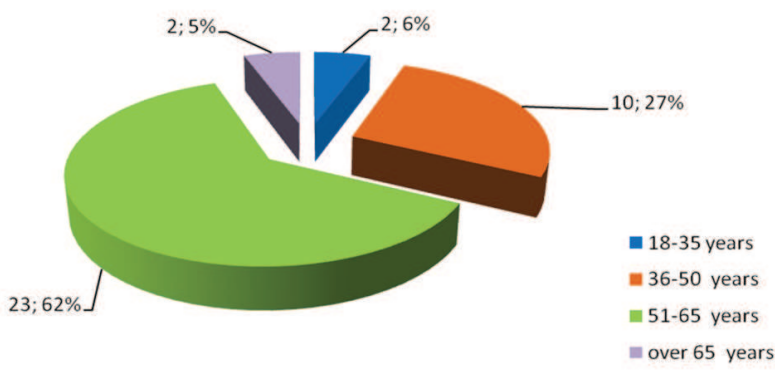

Fig. 2. Division by age study, belongs to the age range 50-65 years. It corresponds to the data for European and world trends in morbidity.

On the question related to comorbidity, 11 (or $30 \%$ ) of the patients have responded that they do not have accompanying diseases and 26 (or 70\%) have more than one concomitant disease (two or more).

On the question whether they have insomnia, 20 (or 54\%) of women gave a positive answer and 17 (or 46\%) denied. Regarding the question if there is a change in their attitude towards the surroundings (Fig. 3), 17\% (or 46\%) confirmed and 20 (or 54\%) denied the statement.

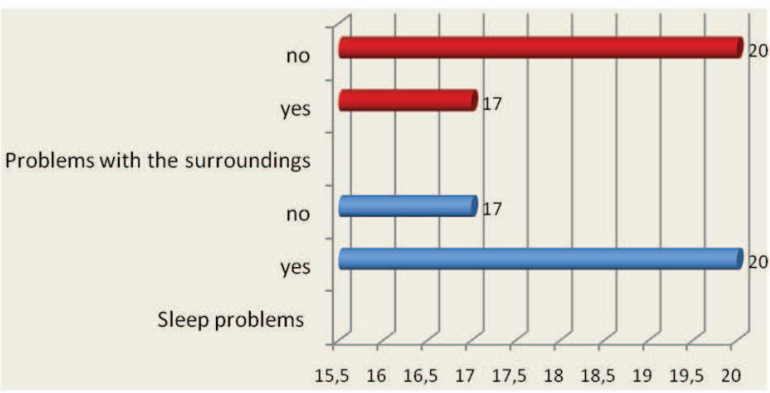

Fig. 3. Problems with sleep and the acceptance of the surroundings (number of individuals)

The freely structured inquiry contains a question asking women to evaluate their level of anxiety according to a 0 to 10 scale (Fig. 4). Although the responses look heterogeneous at first glance, they give the impression that a total of 33 persons (or 89\%) pointed out average and above the average levels of anxiety. 4 women only (or 11\%) have a self-assesed anxiety below the avarage scale.

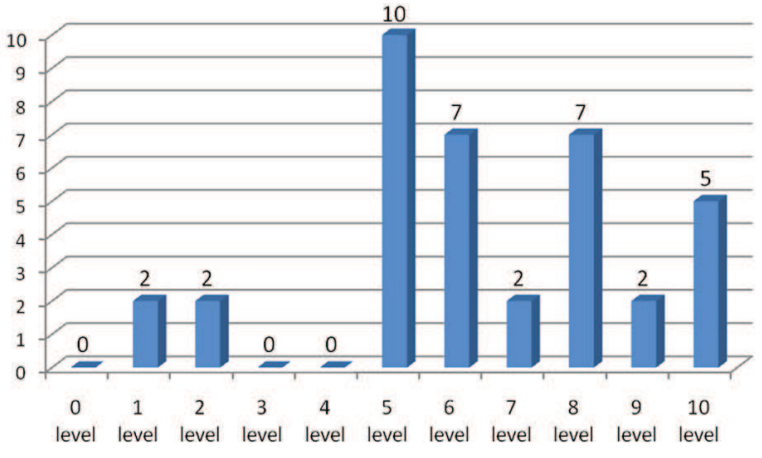

Fig. 4. Number of women and their anxiety levels 
Silviya Tzvetkova, Kameliya Tsvetanova, Ivelina Ivanova et al.

There is a predominance of higher levels of selfassessed anxiety in the patients inquired.

On the question about sexual problems (Fig. 5), e.g. whether their sexual life has changed after the cancer diagnose, the majority of the patients 16 (or 43\%) responds that they have problems in their sexual life, 13 (or 35\%) respond they do not have such problems and 7 (or 19\%) report partial changes. One woman only (or 3\%) has not replied to the question. So changes in the sexual relations are recorded in 27 (or 73\%) female patients. Sexual life changes as a result of the diagnosis.

We assume the possibility part of the negative responses to be influenced by nation-psychological specifics, deeply connected with the belief that this topic is too intimate to be discussed.

The sexual sphere is very emotional. The problems there do not lead to the meeting of this kind of emotional-physical needs which further increases the level of anxiety.

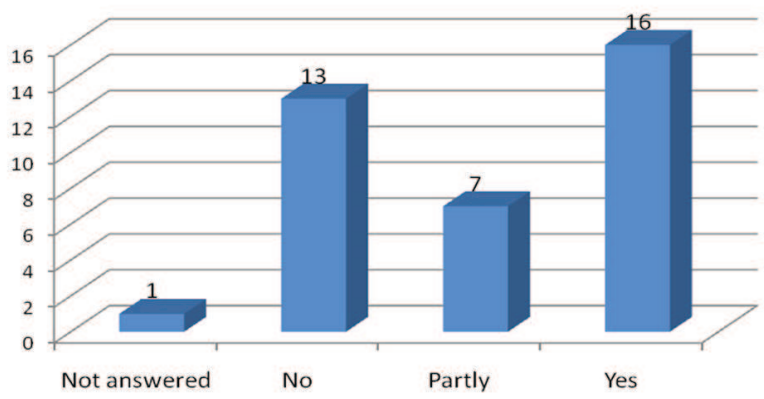

Fig. 5. Sexual problems (number of patients)

Studying the family status of women, it was established that the largest share is held by married women - 26 (or 70\%).

After an analysis of the results of the questionnaire for measurement of anxiety by tools - (Taylor Manifest Anxiety Scale - TMAS), (Fig. 6), it was established that 2 (or 5\%) of the women belong to the low level of anxiety group, 15 (or 41\%) patients belong to the group of medium to low level. The group medium to high level consists of 9 (or 24\%) women and the high level of anxiety group is formed by 11 (or 30\%) women.

Accumulation of the results obtained is recorded in the field of medium to low level of anxiety in women. Following in number are the patients with

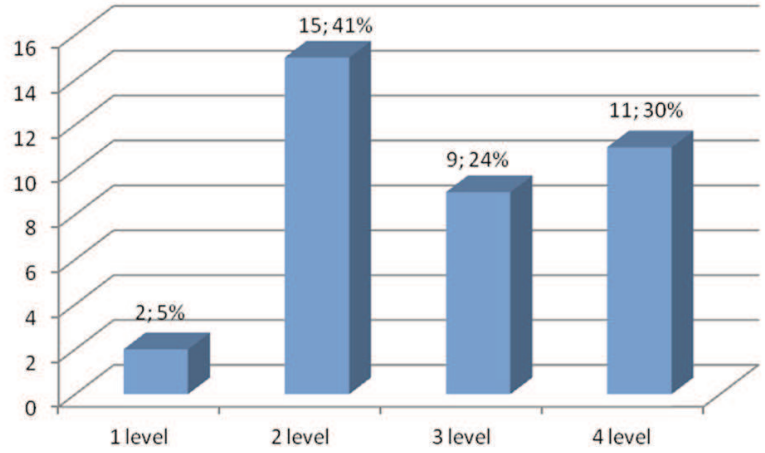

Fig. 6. Anxiety (level) - Taylor Manifest Anxiety Scale

high level of anxiety. Small number is reported of women in the groups with low level and very high level of anxiety. Answers from medium to high level of anxiety prevail - 20 persons (or $54 \%$ ).

A difference exists between the self-assessed and calculated by tools anxiety of the respondents. In the first case 10 (or 27\%) women who stated medium level of anxiety are registered while there are 15 (or 41\%) in the second case. In freely structured query the patient chooses and controls himself the result based on a scale from 0 to 10 , while the result according to the second method is dormant (obtained after processing of the results). Similar conclusions can be made regarding the number of inquired patients with levels of anxiety above the average: from the free questions it can be seen that 23 (or 62\%) belong to this group and from the Taylor's methodology - 20 (or 54\%) women fall into this group. Due to the limited number of women with breast cancer inquired, the differences in the results discussed cannot be considered statistically significant but they give a direction for searching an explanation in the specificity of the tools and their internal processing by patients.

Examining the emotional changes through the Distress thermometer and EORTC QLQ-C30 it was proven (Table 1) that according to the first methodology, the percentage is higher of women who describe themselves as more nervous, sad, anxious, angry or afraid.

According to EORTC the sum of items which build the functional scale „emotionality”, namely strenuousness, anxiety, irritability and depression, is determined at four levels: low, medium, high and very high. As it can be seen from Table 1, a total of 26 
women (or 70\%) belong to the parameter from medium to very high level of the emotional changes.

Table 1. Emotional changes according to Distress thermometer and EORTC QLQ-C30

\begin{tabular}{|c|c|c|c|}
\hline & Indicators & In number & $\%$ \\
\hline \multirow{7}{*}{ 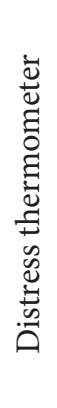 } & depression & 6 & 10 \\
\hline & fear & 9 & 15 \\
\hline & nervousness & 14 & 24 \\
\hline & sadness & 13 & 22 \\
\hline & anxiety/anger & 9 & 15 \\
\hline & $\begin{array}{l}\text { a lot of interest towards usual } \\
\text { activities }\end{array}$ & 3 & 5 \\
\hline & no desire for making plans & 5 & 9 \\
\hline \multirow{5}{*}{ 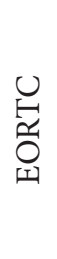 } & emotional functioning - score & & \\
\hline & under 4 & 9 & 24 \\
\hline & from 5 to 9 & 19 & 51 \\
\hline & from 10 to 14 & 5 & 14 \\
\hline & over 15 & 2 & 5 \\
\hline
\end{tabular}

Some conclusions can be made from the statistical processing (Multiple-Variable Analysis, Spearman Rang Correlations):

$\diamond$ Trying to find interrelationship between the demographic indicators and indicators that determine emotional changes, a correlation was found between age, place of living and duration of disease $(\mathrm{r}=0.33, \mathrm{p}=0.05)$. Emotional changes do not depend on education, employment and number of operations undergone. A reverse correlation was established by EORTC QLQ$\mathrm{C} 30$ between the family status and the item for specifying anxiety $(\mathrm{r}=-0.41, \mathrm{p}=0.02)$;

$\diamond$ There is a significant connection between selfassessed level of anxiety (by Freely structured inquiry) according to the scale $0-10$ and indicators for emotional problems of the EORTC methodology: with the question measuring strenuousness - $\mathrm{r}=0.47, \mathrm{p}=0.005$, the question measuring anxiety - $r=0.41, p=0.02$; the question measuring irritability - $\mathrm{r}=0.41, \mathrm{p}=0.02$; the question measuring depression - $\mathrm{r}=0.46$, $\mathrm{p}=0.01$;

$\diamond$ Statistically significant correlation was established between self-assessed (freely structured inquiry) and calculated by means of tools level of anxiety (TMAS) when $\mathrm{r}=0.55, \mathrm{p}=0.002$;

$\diamond$ Significant statistical relation exists between the items of EORTC QLQ-C30: strenuousness, anxiety, and irritability (when $\mathrm{r}=0.52$ to 0.58 , $\mathrm{p}=0.001$ to 0.003 );

$\diamond$ The indicator depression is in significant correlation with strenuousness $(\mathrm{r}=0.54, \mathrm{p}=0.002)$, anxiety $(\mathrm{r}=0.76, \mathrm{p}=0.000)$ and irritability $(\mathrm{r}=0.61, \mathrm{p}=0.000)$ as well as with anxiety levels of the TMAS methodology when $\mathrm{r}=0.62$, $\mathrm{p}=0.005$.

In a research study conducted before among 75 oncological patients (22, c. $569-579)$, it was determined that 49 (or 65\%) do not like to share their emotions and thoughts with their relatives and 52 (or $69 \%$ ) are anxious about the effects when others come to know their diagnosis. 47 (63\%) fear abandonment and loneliness. The patients demonstrate in different ways their anxiety during the inquiry. Higher educated patients are inclined to have controlled anxiety and stress (Crostabulation Analysis, Cramer $=0.47$, $\mathrm{p}=0.002)$.

Own researches conducted by the present moment (among nearly 160 persons), partly related to the issue of anxiety in cancer female patients indicate that the idea and the depth of understanding of the diagnosis are connected with basic personal structures, assumed to be relatively constant but open to a different extent to crises and/or assert. Cognitive schemes for processing the information, working till the moment of diagnosing prove to be inefficient subsequently, as they are strongly influenced by the dynamic changes in emotional, psychic and social spheres.

\section{CONCLUSION}

In the conducted study, specifics and dependencies, which were related to the emotional functioning in women with breast cancer were established such as deepening of the emotional changes associated with age, place of residence and duration of illness. However, they do not depend on education and employment. Living conditions in full and extended monocular family are associated with lower levels of anxiety and distress among the investigated persons. 
Silviya Tzvetkova, Kameliya Tsvetanova, Ivelina Ivanova et al.

All used methods demonstrate accumulation of results from average to very high levels of anxiety (free-structured questionnaire, distress thermometer, TMAS and EORTC).

In a study of emotional changes using the Distress-thermometer, it was established that most of the women define themselves as nervous, sad, anxious, angry and frightened.

Changes in the emotional sphere, indicating anxiety were significantly positively correlated with conditions such as anxiety, depression and irritability - which leads to the conclusion that reducing the irritability, the depression and the tension will decrease the personal and the situational anxiety.

Breast cancer is one of the leading diseases in the modern society. It takes place among the socially significant diseases. The cancer of the mammary gland is important and avoidable reason for death, with the implementation of effective screening programmes for early detection and treatment of these cases. On the basis of a startling statistics, a need arises of early prophylaxis, especially for women from risk groups and bearing family history, as well as a need of early diagnostics and timely therapy. By means of the new imaging methods which continuously make their way into everyday practise, an opportunity is there for cancer to be diagnosed and completely cured when detected on time. Thanks to the rapid pace of development of oncology, female patients with such diagnosis have the opportunity to be treated with the latest in the sphere of radiation therapy and therapeutic treatment of cancer. Through the new methods for operational treatment and the reconstruction methods - the so-called oncoplastic approach, the operational interventions are less traumatic and less disabling. So the women feel more complete and feminine.

The changes in one area influence the other areas of operation which proves the complex nature of the concept "quality of life”. Anxiety generates suspiciousness and fear. It reduces the self-evaluation, affects negatively the potential for independent handling of daily activities and for decision making, provokes adjustments for symbiotic behaviour, reduces emotional and volitional characteristics and questions the achievements.
The disease inevitably leaves a trace in women and mostly changes their quality of life, it influences their psychosocial condition, causes anxiety and distress and changes their views on life and future. The emotional condition and intellectual capabilities have a key importance in the perception of the cancer disease. Encapsulation and emotional-social problems are some of the challenges before the team of specialists treating these women.

One of the most successful communication approaches aiming to help these female patients is the establishment of an atmosphere of trust where they can improve their ability and willingness for selfcontrol in the process of treatment, in decision making and communication. Consultations with psychologists are extremely necessary (18) to limit the alarming symptoms, distress and self-isolation.

The progress in the treatment of breast cancer is achieved through the successful implementation of a multidisciplinary approach, personalized for the individual patient, so that the negative effects of the disease process on the personality to be minimized.

\section{DECLARATION OF INTEREST}

None.

No conflict of interest.

\section{REFERENCES}

1. Baychev G. Diseases of the mammary gland. Sofia, 2013, Spectrum AD, p. 126, 173, 176-177.

2. Breast cancer - statistics. nmgenomix.com, 14.12.2015.

3. Ganz P., Hirji K., Sim M., Schag C., Fred C. \& Polinsky M. Predicting psychosocial risk in patients with breast cancer. Medical Care, 31(5), 1993, 419-431.

4. Sarna L. Women with lung cancer: Impact on quality of life. Quality of Life Research, 2(1), 1993, 13-22.

5. Levine $S$. The changing terrain of medical sociology: Emergent concern with quality of life. Journal of Health and Social Behaviour, 28(1), 1987, 1-7.

6. Ganeva, Z. Another thing about the breast cancer. Sofia, 2013, Elestra, p.82-87, p.89-90.

7. Van Halteren H. Rehabilitation issues during cancer treatment and follow up, European Society for Medical Oncology, UK, 2014. 
8. International Agency for Research on Cancer. Breast cancer. Estimated incidence from breast cancer in women, 2012, www.iarc.fr, 14.12.2015.

9. www. iarc.fr. 2015

10. Andersen, B., Anderson, B. \& deProsse, C. Controlled prospective longitudinal study of women with cancer: II. Psychological outcomes. Journal of Consulting and Clinical Psychology, 1989, 57, 692-697.

11. Ganz P., Coscarelli, C., Fred, C., Kahn, B., Polinsky, M., \& Petersen, L. (Breast cancer survivors: Psychosocial concerns and quality of life. Breast Cancer Research and Treatment, 1996, 38, 183-199.

12. Dorval M., Maunsell E., Deschenes, L., Brisson J., \& Masse B. Long-term quality of life after breast cancer: Comparison of 8-year survivors with population controls. Journal of Clinical Oncology, 1998, $16,487-494$.

13. Sorenson M. Psychosocial factors in quality of life for long-term survivors of breast cancer and their spouses. Dissertation Abstracts Int (B) 55, 1994, 607.

14. Kalev D and editors. Clinical behaviour in case of breast carcinoma, Varna, 2013, p.189-191.

15. Barraclough, J. Cancer and emotion: A practical guide to psycho-oncology. Chichester: John Wiley \& Sons Limited, 1999.

16. Osteen R., Cady B., Friedman M., Kraybill W., Doggett S., Hussey D., Urist M., Chmiel J., Clive R., $\&$ Winchester D. Patterns of care for younger women with breast cancer. Journal of National Cancer Institute Monographs. 16, 1994, 43-46.

17. Shapiro D. And in Health: A Guide for Couples Facing Cancer Together. Trumpeter/Random House, 2013.

18. Tsvetkova S., D. Yulieva, I. Petkova. Need of psychological consultation in the treatment of cancer patients. Applied Psychology and social practise: possibilities and perspectives, Varna, 2014, p. 569-579.

19. Ivanov I. Methodologies for examination of functional conditions. Shumen, 1999, p.11.

20. Tsvetkova S., D. Kermova. Stresses in the psychological-consultation work with post-operation cancer patients, The Applied Psychology in Bulgaria: possibilities and perspectives 2012, Varna, p. 536-547. 